\title{
Information Geometry and Chaos on Negatively Curved Statistical Manifolds
}

\author{
Carlo Cafard* \\ Department of Physics, \\ State University of New York at Albany-SUNY,1400 Washington \\ Avenue, Albany, NY 12222, USA
}

\begin{abstract}
A novel information-geometric approach to chaotic dynamics on curved statistical manifolds based on Entropic Dynamics (ED) is suggested. Furthermore, an information-geometric analogue of the Zurek-Paz quantum chaos criterion is proposed. It is shown that the hyperbolicity of a nonmaximally symmetric $6 N$-dimensional statistical manifold $\mathcal{M}_{s}$ underlying an ED Gaussian model describing an arbitrary system of $3 N$ non-interacting degrees of freedom leads to linear informationgeometric entropy growth and to exponential divergence of the Jacobi vector field intensity, quantum and classical features of chaos respectively.
\end{abstract}

PACS numbers: $02.50 . \mathrm{Tt}, 02.50 . \mathrm{Cw}, 02.40 .-\mathrm{k}, 05.45 .-\mathrm{a}$

Keywords: Inductive inference, information geometry, statistical manifolds, entropy, nonlinear dynamics and chaos.

\section{INTRODUCTION}

Entropic Dynamics (ED) [1], namely the combination of principles of inductive inference (Maximum relative Entropy Methods, [2]) and Information Geometry (IG) [3], is a theoretical framework constructed on statistical manifolds and it is developed to investigate the possibility that laws of physics, either classical or quantum, might reflect laws of inference rather than laws of nature. This paper is a follow up of a series of the author's works $[4,5]$. In this article, we use the ED theoretical framework to explore the possibility of constructing a unifying (classical and quantum) criterion of chaos. We assume the system under investigation has $3 N$ degrees of freedom, each one described by two pieces of relevant information, its expectation value and its variance (Gaussian statistical variables). This leads to consider an ED model on a non-maximally symmetric $6 N$-dimensional statistical manifold $\mathcal{M}_{s}$. The manifold $\mathcal{M}_{s}$ has constant negative Ricci curvature proportional to the number of degrees of freedom of the system, $R_{\mathcal{M}_{s}}=-3 N$. An information-geometric analog of the Zurek-Paz quantum chaos criterion is suggested. It is shown that the system explores statistical volume elements on $\mathcal{M}_{s}$ at an exponential rate. We define a dynamical informationgeometric entropy $S_{\mathcal{M}_{s}}$ of the system and we show it increases linearly in time (statistical evolution parameter) and is proportional to the number of degrees of freedom of the system. The geodesics on $\mathcal{M}_{s}$ are hyperbolic trajectories. Using the Jacobi-Levi-Civita (JLC) equation for geodesic spread, it is shown that the Jacobi vector field intensity $J_{\mathcal{M}_{s}}$ diverges exponentially and is proportional to the number of degrees of freedom of the system. Thus, $R_{\mathcal{M}_{s}}, S_{\mathcal{M}_{s}}$ and $J_{\mathcal{M}_{s}}$ are proportional to the number of Gaussian-distributed microstates of the system. This proportionality leads to conclude there exists a substantial link among these information-geometric indicators of chaoticity.

\section{THE ED GAUSSIAN MODEL}

Given two probability distributions, how can one define a notion of "distance" between them? The answer to this question is provided by IG. As it is shown in [6] and [7], the notion of distance between dissimilar probability distributions is quantified by the Fisher-Rao information metric tensor. We consider an ED model whose microstates span a $3 N$-dimensional space labelled by the variables $\{\vec{X}\}=\left\{\vec{x}^{(1)}, \vec{x}^{(2)}, \ldots ., \vec{x}^{(N)}\right\}$ with $\vec{x}^{(\alpha)} \equiv\left(x_{1}^{(\alpha)}, x_{2}^{(\alpha)}, x_{3}^{(\alpha)}\right)$, $\alpha=1, \ldots, N$ and $x_{a}^{(\alpha)} \in \mathbb{R}$ with $a=1,2,3$. We assume the only testable information pertaining to the quantities $x_{a}^{(\alpha)}$ consists of the expectation values $\left\langle x_{a}^{(\alpha)}\right\rangle$ and the variance $\Delta x_{a}^{(\alpha)}=\sqrt{\left\langle\left(x_{a}^{(\alpha)}-\left\langle x_{a}^{(\alpha)}\right\rangle\right)^{2}\right\rangle}$. The set of these expected values define the $6 N$-dimensional space of macrostates of the system. A measure of distinguishability among the states of the ED model is achieved by assigning a probability distribution $P(\vec{X} \mid \vec{\Theta})$ to each macrostate $\vec{\Theta}$ where

*Electronic address: carlocafaro2000@yahoo.it 
$\{\vec{\Theta}\}=\left\{{ }^{(1)} \theta_{a}^{(\alpha)},{ }^{(2)} \theta_{a}^{(\alpha)}\right\}$ with $\alpha=1,2, \ldots ., N$ and $a=1,2,3$. The process of assigning a probability distribution to each state provides $\mathcal{M}_{S}$ with a metric structure. Specifically, the Fisher-Rao information metric defined in (7) is a measure of distinguishability among macrostates. It assigns an IG to the space of states.

\section{THE STATISTICAL MANIFOLD $\mathcal{M}_{S}$}

Consider an arbitrary physical system evolving over a $3 N$-dimensional space. The variables $\{\vec{X}\}=$ $\left\{\vec{x}^{(1)}, \vec{x}^{(2)}, \ldots ., \vec{x}^{(N)}\right\}$ label the $3 N$-dimensional space of microstates of the system. Each macrostate may be thought as a point of a $6 \mathrm{~N}$-dimensional statistical manifold with coordinates given by the numerical values of the expectations ${ }^{(1)} \theta_{a}^{(\alpha)}=\left\langle x_{a}^{(\alpha)}\right\rangle$ and ${ }^{(2)} \theta_{a}^{(\alpha)}=\Delta x_{a}^{(\alpha)} \equiv \sqrt{\left\langle\left(x_{a}^{(\alpha)}-\left\langle x_{a}^{(\alpha)}\right\rangle\right)^{2}\right\rangle}$. The available information can be written in the form of the following $6 \mathrm{~N}$ information constraint equations,

$$
\begin{gathered}
\left\langle x_{a}^{(\alpha)}\right\rangle=\int_{-\infty}^{+\infty} d x_{a}^{(\alpha)} x_{a}^{(\alpha)} P_{a}^{(\alpha)}\left(x_{a}^{(\alpha)} \mid(1) \theta_{a}^{(\alpha)},{ }^{(2)} \theta_{a}^{(\alpha)}\right) \\
\Delta x_{a}^{(\alpha)}=\left[\int_{-\infty}^{+\infty} d x_{a}^{(\alpha)}\left(x_{a}^{(\alpha)}-\left\langle x_{a}^{(\alpha)}\right\rangle\right)^{2} P_{a}^{(\alpha)}\left(x_{a}^{(\alpha)} \mid(1) \theta_{a}^{(\alpha)},{ }^{(2)} \theta_{a}^{(\alpha)}\right)\right]^{\frac{1}{2}}
\end{gathered}
$$

where ${ }^{(1)} \theta_{a}^{(\alpha)}=\left\langle x_{a}^{(\alpha)}\right\rangle$ and ${ }^{(2)} \theta_{a}^{(\alpha)}=\Delta x_{a}^{(\alpha)}$ with $\alpha=1,2, \ldots, N$ and $a=1,2,3$. The probability distributions $P_{a}^{(\alpha)}$ are constrained by the conditions of normalization,

$$
\int_{-\infty}^{+\infty} d x_{a}^{(\alpha)} P_{a}^{(\alpha)}\left(x_{a}^{(\alpha)} \mid(1) \theta_{a}^{(\alpha)},{ }^{(2)} \theta_{a}^{(\alpha)}\right)=1 .
$$

Information theory identifies the Gaussian distribution as the maximum entropy distribution if only the expectation value and the variance are known. The distribution that best reflects the information contained in the prior distribution $m(\vec{X})$ updated by the information $\left(\left\langle x_{a}^{(\alpha)}\right\rangle, \Delta x_{a}^{(\alpha)}\right)$ is obtained by maximizing the relative entropy

$$
S(\vec{\Theta})=-\int d^{3 N} \vec{X} P(\vec{X} \mid \vec{\Theta}) \log \left(\frac{P(\vec{X} \mid \vec{\Theta})}{m(\vec{X})}\right),
$$

where $m(\vec{X})$ is the prior probability distribution. As a working hypothesis, the prior $m(\vec{X})$ is set to be uniform since we assume the lack of prior available information about the system (postulate of equal a priori probabilities). Upon maximizing (3), given the constraints (1) and (2), we obtain

$$
P(\vec{X} \mid \vec{\Theta})=\prod_{\alpha=1}^{N} \prod_{a=1}^{3} P_{a}^{(\alpha)}\left(x_{a}^{(\alpha)} \mid \mu_{a}^{(\alpha)}, \sigma_{a}^{(\alpha)}\right)
$$

where

$$
P_{a}^{(\alpha)}\left(x_{a}^{(\alpha)} \mid \mu_{a}^{(\alpha)}, \sigma_{a}^{(\alpha)}\right)=\left(2 \pi\left[\sigma_{a}^{(\alpha)}\right]^{2}\right)^{-\frac{1}{2}} \exp \left[-\frac{\left(x_{a}^{(\alpha)}-\mu_{a}^{(\alpha)}\right)^{2}}{2\left(\sigma_{a}^{(\alpha)}\right)^{2}}\right]
$$

and ${ }^{(1)} \theta_{a}^{(\alpha)}=\mu_{a}^{(\alpha)},{ }^{(2)} \theta_{a}^{(\alpha)}=\sigma_{a}^{(\alpha)}$. The probability distribution (4) encodes the available information concerning the system. Note that we have assumed uncoupled constraints among microvariables $x_{a}^{(\alpha)}$. In other words, we assumed that information about correlations between the microvariables need not to be tracked. This assumption leads to the 
simplified product rule (4). However, coupled constraints would lead to a generalized product rule in (4) and to a metric tensor (7) with non-trivial off-diagonal elements (covariance terms). Such generalizations would require more delicate analysis. Deviations from Gaussian-type information constraints and the presence of a nonvanishing correlation coefficient $\left(r=\frac{\left\langle\left(x_{1}-\mu_{1}\right)\left(x_{2}-\mu_{2}\right)\right\rangle}{\sigma_{1} \sigma_{2}}, r\right.$ is the correlation coefficient of the two dependent random variables $x_{1}$ and $\left.x_{2}\right)$ among the random microvariables $x_{a}^{(\alpha)}$ are some of the new topics appearing in a forthcoming paper [8].

\section{A. METRIC STRUCTURE OF $\mathcal{M}_{S}$}

The dimensionless line element $d s$ between $P(\vec{X} \mid \vec{\Theta})$ and $P(\vec{X} \mid \vec{\Theta}+d \vec{\Theta})$ is given by,

$$
d s^{2}=g_{\mu \nu} d \Theta^{\mu} d \Theta^{\nu}
$$

where

$$
g_{\mu \nu}=\int d \vec{X} P(\vec{X} \mid \vec{\Theta}) \frac{\partial \log P(\vec{X} \mid \vec{\Theta})}{\partial \Theta^{\mu}} \frac{\partial \log P(\vec{X} \mid \vec{\Theta})}{\partial \Theta^{\nu}}
$$

is the Fisher-Rao metric. Substituting (4) into (7), the metric $g_{\mu \nu}$ on $\mathcal{M}_{s}$ becomes a $6 N \times 6 N$ matrix $M$ made up of $3 N$ blocks $M_{2 \times 2}$ with dimension $2 \times 2$ given by,

$$
M_{2 \times 2}=\left(\begin{array}{cc}
\left(\sigma_{a}^{(\alpha)}\right)^{-2} & 0 \\
0 & 2 \times\left(\sigma_{a}^{(\alpha)}\right)^{-2}
\end{array}\right)
$$

with $\alpha=1,2, \ldots, N$ and $a=1,2,3$. From (7), the "length" element (6) reads,

$$
d s^{2}=\sum_{\alpha=1}^{N} \sum_{a=1}^{3}\left[\frac{1}{\left(\sigma_{a}^{(\alpha)}\right)^{2}} d \mu_{a}^{(\alpha) 2}+\frac{2}{\left(\sigma_{a}^{(\alpha)}\right)^{2}} d \sigma_{a}^{(\alpha) 2}\right] .
$$

We bring attention to the fact that the metric structure of $\mathcal{M}_{s}$ is an emergent (not fundamental) structure. It arises only after assigning a probability distribution $P(\vec{X} \mid \vec{\Theta})$ to each state $\vec{\Theta}$.

\section{B. CURVATURE OF $\mathcal{M}_{s}$}

The Ricci scalar curvature $R$ is given by,

$$
R=g^{\mu \nu} R_{\mu \nu}
$$

where $g^{\mu \nu} g_{\nu \rho}=\delta_{\rho}^{\mu}$ so that $g^{\mu \nu}=\left(g_{\mu \nu}\right)^{-1}$. The Ricci tensor $R_{\mu \nu}$ is given by,

$$
R_{\mu \nu}=\partial_{\varepsilon} \Gamma_{\mu \nu}^{\varepsilon}-\partial_{\nu} \Gamma_{\mu \varepsilon}^{\varepsilon}+\Gamma_{\mu \nu}^{\varepsilon} \Gamma_{\varepsilon \eta}^{\eta}-\Gamma_{\mu \varepsilon}^{\eta} \Gamma_{\nu \eta}^{\varepsilon} .
$$

The Christoffel symbols $\Gamma_{\mu \nu}^{\rho}$ appearing in the Ricci tensor are defined in the standard way,

$$
\Gamma_{\mu \nu}^{\rho}=\frac{1}{2} g^{\rho \varepsilon}\left(\partial_{\mu} g_{\varepsilon \nu}+\partial_{\nu} g_{\mu \varepsilon}-\partial_{\varepsilon} g_{\mu \nu}\right)
$$

Using (9) and the definitions given above, we can show that the Ricci scalar curvature becomes

$$
R_{\mathcal{M}_{s}}=-3 N<0
$$

From (13) we conclude that $\mathcal{M}_{s}$ is a $6 N$-dimensional statistical manifold of constant negative Ricci scalar curvature. A detailed analysis on the calculation of Christoffel connection coefficients using the ED formalism for a four-dimensional manifold of Gaussians can be found in [5]. Furthermore, it can be shown that $\mathcal{M}_{s}$ is not a pseudosphere (maximally symmetric manifold) since its sectional curvature is not constant. Considerations about the negativity of the Ricci curvature as a strong criterion of dynamical instability and the necessity of compactness of $\mathcal{M}_{s}$ in "true" chaotic dynamical systems will appear in [8]. 


\section{CANONICAL FORMALISM FOR THE ED-GAUSSIAN MODEL}

At this point, we study the trajectories of the system on $\mathcal{M}_{s}$. We emphasize ED can be derived from a standard principle of least action (Maupertuis- Euler-Lagrange-Jacobi-type) [1, 9]. The geodesic equations for the macrovariables of the Gaussian ED model are given by,

$$
\frac{d^{2} \Theta^{\mu}}{d \tau^{2}}+\Gamma_{\nu \rho}^{\mu} \frac{d \Theta^{\nu}}{d \tau} \frac{d \Theta^{\rho}}{d \tau}=0
$$

with $\mu=1,2, \ldots, 6 N$. Observe that the geodesic equations are nonlinear, second order coupled ordinary differential equations.

\section{A. GEODESICS ON $\mathcal{M}_{s}$}

We seek the explicit form of (14) for the pairs of statistical coordinates $\left(\mu_{a}^{(\alpha)}, \sigma_{a}^{(\alpha)}\right)$. Substituting the explicit expression of the Christoffel connection coefficients into (14), the geodesic equations for the macrovariables $\mu_{a}^{(\alpha)}$ and $\sigma_{a}^{(\alpha)}$ associated to the microstate $x_{a}^{(\alpha)}$ become,

$$
\frac{d^{2} \mu_{a}^{(\alpha)}}{d \tau^{2}}-\frac{2}{\sigma_{a}^{(\alpha)}} \frac{d \mu_{a}^{(\alpha)}}{d \tau} \frac{d \sigma_{a}^{(\alpha)}}{d \tau}=0, \frac{d^{2} \sigma_{a}^{(\alpha)}}{d \tau^{2}}-\frac{1}{\sigma_{a}^{(\alpha)}}\left(\frac{d \sigma_{a}^{(\alpha)}}{d \tau}\right)^{2}+\frac{1}{2 \sigma_{a}^{(\alpha)}}\left(\frac{d \mu_{a}^{(\alpha)}}{d \tau}\right)^{2}=0 .
$$

with $\alpha=1,2, \ldots, N$ and $a=1,2,3$. This is a set of coupled ordinary differential equations, whose solutions are

$$
\begin{gathered}
\mu_{a}^{(\alpha)}(\tau)=\frac{\left(B_{a}^{(\alpha)}\right)^{2}}{2 \beta_{a}^{(\alpha)}} \frac{1}{\cosh \left(2 \beta_{a}^{(\alpha)} \tau\right)-\sinh \left(2 \beta_{a}^{(\alpha)} \tau\right)+\frac{\left(B_{a}^{(\alpha)}\right)^{2}}{8\left(\beta_{a}^{(\alpha)}\right)^{2}}}+C_{a}^{(\alpha)} \\
\sigma_{a}^{(\alpha)}(\tau)=B_{a}^{(\alpha)} \frac{\cosh \left(\beta_{a}^{(\alpha)} \tau\right)-\sinh \left(\beta_{a}^{(\alpha)} \tau\right)}{\cosh \left(2 \beta_{a}^{(\alpha)} \tau\right)-\sinh \left(2 \beta_{a}^{(\alpha)} \tau\right)+\frac{\left(B_{a}^{(\alpha)}\right)^{2}}{8\left(\beta_{a}^{(\alpha)}\right)^{2}}}
\end{gathered}
$$

The quantities $B_{a}^{(\alpha)}, C_{a}^{(\alpha)}, \beta_{a}^{(\alpha)}$ are real integration constants and they can be evaluated once the boundary conditions are specified. We are interested in investigating the stability of the trajectories of the ED model considered on $\mathcal{M}_{s}$. It is known [9] that the Riemannian curvature of a manifold is closely connected with the behavior of the geodesics on it. If the Riemannian curvature of a manifold is negative, geodesics (initially parallel) rapidly diverge from one another. For the sake of simplicity, we assume very special initial conditions: $B_{a}^{(\alpha)} \equiv \Lambda, \beta_{a}^{(\alpha)} \equiv \lambda \in \mathbb{R}^{+}, C_{a}^{(\alpha)}=0$, $\forall \alpha=1,2, \ldots, N$ and $a=1,2,3$. However, the conclusion we reach can be generalized to more arbitrary initial conditions. It is worthwhile noticing that, in our case, $\mathcal{M}_{s}$ is a geodesically complete manifold since every maximal geodesic is well-defined for all temporal parameters $\tau$. Therefore, $\mathcal{M}_{s}$ represents a natural setting for global questions in this Riemannian geometric framework applied to probability theory and the search for a weak criterion of chaos can be carried out [8].

\section{LINEARITY OF THE INFORMATION-GEOMETRIC DYNAMICAL ENTROPY}

Recall that $\mathcal{M}_{s}$ is the space of probability distributions $P(\vec{X} \mid \vec{\Theta})$ labeled by $6 N$ statistical parameters $\vec{\Theta}$. These parameters are the coordinates for the point $P$, and in these coordinates a volume element $d V_{\mathcal{M}_{s}}$ reads,

$$
d V_{\mathcal{M}_{S}}=\sqrt{g} d^{6 N} \vec{\Theta}=\prod_{\alpha=1}^{N} \prod_{a=1}^{3} \frac{\sqrt{2}}{\left(\sigma_{a}^{(\alpha)}\right)^{2}} d \mu_{a}^{(\alpha)} d \sigma_{a}^{(\alpha)}
$$

The volume of an extended region $\Delta V_{\mathcal{M}_{s}}(\tau ; \lambda)$ of $\mathcal{M}_{s}$ is defined by,

$$
\Delta V_{\mathcal{M}_{s}}(\tau ; \lambda) \stackrel{\text { def }}{=} \prod_{\alpha=1}^{N} \prod_{a=1}^{3} \int_{\mu_{a}^{(\alpha)}(0)}^{\mu_{a}^{(\alpha)}(\tau)} \int_{\sigma_{a}^{(\alpha)}(0)}^{\sigma_{a}^{(\alpha)}(\tau)} \frac{\sqrt{2}}{\left(\sigma_{a}^{(\alpha)}\right)^{2}} d \mu_{a}^{(\alpha)} d \sigma_{a}^{(\alpha)}
$$


where $\mu_{a}^{(\alpha)}(\tau)$ and $\sigma_{a}^{(\alpha)}(\tau)$ are given in (16). The quantity that encodes relevant information about the stability of neighboring volume elements is the the average volume $\bar{V}_{\mathcal{M}_{s}}(\tau ; \lambda)$,

$$
\bar{V}_{\mathcal{M}_{s}}(\tau ; \lambda) \equiv\left\langle\Delta V_{\mathcal{M}_{s}}(\tau ; \lambda)\right\rangle_{\tau} \stackrel{\text { def }}{=} \frac{1}{\tau} \int_{0}^{\tau} \Delta V_{\mathcal{M}_{s}}\left(\tau^{\prime} ; \lambda\right) d \tau^{\prime} \stackrel{\tau \rightarrow \infty}{\approx} e^{3 N \lambda \tau}
$$

This asymptotic regime of diffusive evolution in (19) describes the exponential increase of average volume elements on $\mathcal{M}_{s}$. The exponential instability characteristic of chaos forces the system to rapidly explore large areas (volumes) of the statistical manifolds. It is interesting to note that this asymptotic behavior appears also in the conventional description of quantum chaos where the entropy increases linearly at a rate determined by the Lyapunov exponents. The linear entropy increase as a quantum chaos criterion was introduced by Zurek and Paz [10]. In our informationgeometric approach a relevant variable that can be useful to study the degree of instability characterizing the ED model is the information-geometric entropy quantity defined as,

$$
S_{\mathcal{M}_{s}} \stackrel{\text { def }}{=} \lim _{\tau \rightarrow \infty} \log \bar{V}_{\mathcal{M}_{s}}(\tau ; \lambda) \text {. }
$$

Substituting (18) in (19), equation (20) becomes,

$$
S_{\mathcal{M}_{s}} \stackrel{\tau \rightarrow \infty}{\approx} 3 N \lambda \tau
$$

The entropy-like quantity $S_{\mathcal{M}_{s}}$ in (21) is the asymptotic limit of the natural logarithm of the statistical weight $\left\langle\Delta V_{\mathcal{M}_{s}}\right\rangle_{\tau}$ defined on $\mathcal{M}_{s}$ and it grows linearly in time, a quantum feature of chaos. Indeed, equation (21) may be considered the information-geometric analog of the Zurek-Paz chaos criterion. Zurek and Paz considered a chaotic system, a single unstable harmonic oscillator characterized by a potential $V(x)=-\frac{\lambda x^{2}}{2}(\lambda$ is the Lyapunov exponent), coupled to an external environment. In the reversible classical limit, the von Neumann entropy of such a system increases linearly at a rate determined by the Lyapunov exponent,

$$
S_{\text {quantum }}^{\text {(chatic })}(\text { Zurek-Paz) } \stackrel{\tau \rightarrow \infty}{\sim} \lambda \tau .
$$

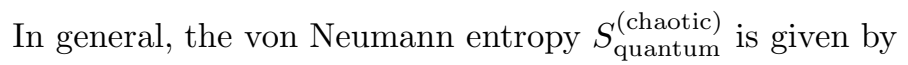

$$
S_{\text {quantum }}^{\text {(chaotic) }}=-\operatorname{tr}\left(\widehat{\rho} \log _{2} \widehat{\rho}\right)=-\sum_{j} \lambda_{j} \log _{2} \lambda_{j}
$$

where the normalized $(\operatorname{tr}(\widehat{\rho})=1)$ density operator $\widehat{\rho}$ is defined as

$$
\widehat{\rho}=\sum_{j} \lambda_{j}\left|\psi_{j}\right\rangle\left\langle\psi_{j}|, \widehat{\rho}| \psi_{j}\right\rangle=\lambda_{j}\left|\psi_{j}\right\rangle .
$$

Notice that the consideration of $3 N$ uncoupled identical unstable harmonic oscillators characterized by potentials $V_{i}(x)=-\frac{\lambda_{i} x^{2}}{2}\left(\lambda_{i}=\lambda_{j} ; i, j=1,2, \ldots, 3 N\right)$ would simply lead to

$$
S_{\text {quantum }}^{\text {(chaotic) }}(\text { Zurek-Paz) } \stackrel{\tau \rightarrow \infty}{\sim} 3 N \lambda \tau .
$$

The resemblance of equations (21) and (25), either in the form or the content is astonishing. A detailed discussion about this result and an additional discussion about a possible connection of (21) to the Kolmogorov-Sinai entropy, one of the most powerful indicators of chaos in classical dynamical systems, will appear in [8].

\section{EXPONENTIAL DIVERGENCE OF THE JACOBI FIELD INTENSITY ON $\mathcal{M}_{s}$}

Finally, we consider the behavior of the one-parameter family of neighboring geodesics $\mathcal{F}_{G_{\mathcal{M}_{s}}}(\lambda) \equiv$ $\left\{\Theta_{\mathcal{M}_{s}}^{\mu}(\tau ; \lambda)\right\}_{\lambda \in \mathbb{R}^{+}}^{\mu=1, ., 6 N}$ where,

$$
\begin{aligned}
& \mu_{a}^{(\alpha)}(\tau ; \lambda)=\frac{\Lambda^{2}}{2 \lambda} \frac{1}{\cosh (2 \lambda \tau)-\sinh (2 \lambda \tau)+\frac{\Lambda^{2}}{8 \lambda^{2}}} \\
& \sigma_{a}^{(\alpha)}(\tau ; \lambda)=\Lambda \frac{\cosh (\lambda \tau)-\sinh (\lambda \tau)}{\cosh (2 \lambda \tau)-\sinh (2 \lambda \tau)+\frac{\Lambda^{2}}{8 \lambda^{2}}}
\end{aligned}
$$


with $\alpha=1,2, \ldots, N$ and $a=1,2,3$. The relative geodesic spread on a (non-maximally symmetric) curved manifold as $\mathcal{M}_{s}$ is characterized by the Jacobi-Levi-Civita equation, the natural tool to tackle dynamical chaos [11, 12],

$$
\frac{D^{2} \delta \Theta^{\mu}}{D \tau^{2}}+R_{\nu \rho \sigma}^{\mu} \frac{\partial \Theta^{\nu}}{\partial \tau} \delta \Theta^{\rho} \frac{\partial \Theta^{\sigma}}{\partial \tau}=0
$$

where the Jacobi vector field $J^{\mu}$ is defined as,

$$
J^{\mu} \equiv \delta \Theta^{\mu} \stackrel{\text { def }}{=} \delta_{\lambda} \Theta^{\mu}=\left(\frac{\partial \Theta^{\mu}(\tau ; \lambda)}{\partial \lambda}\right)_{\tau} \delta \lambda .
$$

Equation (27) forms a system of $6 N$ coupled ordinary differential equations linear in the components of the deviation vector field (28) but nonlinear in derivatives of the metric (7). When the geodesics are neighboring but their relative velocity is arbitrary, the corresponding geodesic deviation equation is the so-called generalized Jacobi equation [13]. Substituting (26) in (27) and neglecting the exponentially decaying terms in $\delta \Theta^{\mu}$ and its derivatives, integration of (27) leads to the following asymptotic expression of the Jacobi vector field intensity,

$$
J_{\mathcal{M}_{S}}=\|J\|=\left(g_{\mu \nu} J^{\mu} J^{\nu}\right)^{\frac{1}{2}} \stackrel{\tau \rightarrow \infty}{\approx} 3 N e^{\lambda \tau}
$$

Further details on the derivation of this result for a four-dimensional statistical manifold are in [5]. We conclude that the geodesic spread on $\mathcal{M}_{s}$ is described by means of an exponentially divergent Jacobi vector field intensity $J_{\mathcal{M}}$, a classical feature of chaos. In our approach the quantity $\lambda_{J}$,

$$
\lambda_{J} \stackrel{\text { def }}{=} \lim _{\tau \rightarrow \infty} \frac{1}{\tau} \ln \left(\frac{\left\|J_{\mathcal{M}_{S}}(\tau)\right\|}{\left\|J_{\mathcal{M}_{S}}(0)\right\|}\right)
$$

would play the role of the conventional Lyapunov exponents. In conclusion, we have shown that,

$$
R_{\mathcal{M}_{s}}=-3 N, S_{\mathcal{M}_{s}} \stackrel{\tau \rightarrow \infty}{\approx} 3 N \lambda \tau, J_{\mathcal{M}_{S}} \stackrel{\tau \rightarrow \infty}{\approx} 3 N e^{\lambda \tau}
$$

The Ricci scalar curvature $R_{\mathcal{M}_{s}}$, the information-geometric entropy $S_{\mathcal{M}_{s}}$ and the Jacobi vector field intensity $J_{\mathcal{M}_{S}}$ are proportional to the number of Gaussian-distributed microstates of the system. This proportionality leads to the conclusion that there exists a substantial link among these information-geometric measures of chaoticity, namely

$$
R_{\mathcal{M}_{s}} \sim S_{\mathcal{M}_{s}} \sim J_{\mathcal{M}_{S}}
$$

Equation (32), together with the information-geometric analog of the Zurek-Paz quantum chaos criterion, equation (21), represent the fundamental results of this work. We believe our theoretical modelling scheme may be used to describe actual systems where transitions from quantum to classical chaos scenario occur, but this will be argued elsewhere [8].

\section{FINAL REMARKS}

In conclusion, a Gaussian ED statistical model has been constructed on a $6 N$-dimensional statistical manifold $\mathcal{M}_{s}$. The macro-coordinates on the manifold are represented by the expectation values of microvariables associated with Gaussian distributions. The geometric structure of $\mathcal{M}_{s}$ was studied. The manifold $\mathcal{M}_{s}$ is a curved manifold of constant negative Ricci curvature $-3 N$. The geodesics of the ED model are hyperbolic curves on $\mathcal{M}_{s}$. A study of the stability of geodesics on $\mathcal{M}_{s}$ was presented. The notion of statistical volume elements was introduced to investigate the asymptotic behavior of a one-parameter family of neighboring volumes $\mathcal{F}_{V_{\mathcal{M}_{s}}}(\lambda) \equiv\left\{V_{\mathcal{M}_{s}}(\tau ; \lambda)\right\}_{\lambda \in \mathbb{R}^{+}}$. An information-geometric analog of the Zurek-Paz chaos criterion was suggested. It was shown that the behavior of geodesics is characterized by exponential instability that leads to chaotic scenarios on the curved statistical manifold. These conclusions are supported by a study based on the geodesic deviation equations and on the asymptotic behavior of the Jacobi vector field intensity $J_{\mathcal{M}_{s}}$ on $\mathcal{M}_{s}$. A Lyapunov exponent analog similar to that appearing in the Riemannian geometric approach to chaos [14] was suggested as an indicator of chaoticity. We think this is a relevant result since a rigorous relation among curvature, Lyapunov exponents and Kolmogorov-Sinay entropy is still under investigation [15] and since there does not exist a well defined unifying characterization of chaos in classical and quantum physics due to fundamental differences between the two theories [16]. 


\section{ACKNOWLEDGEMENT}

The author is grateful to Dr. Saleem Ali and Adom Giffin for very useful comments and suggestions. Special thanks go to Prof. Ariel Caticha for clarifying explanations on "Entropic Dynamics" and for his constant support and advice during this work.

\section{REFERENCES}

1. A. Caticha, "Entropic Dynamics", AIP Conf. Proc. 617, 302 (2002).

2. A. Caticha and A. Giffin, "Updating Probabilities", AIP Conf. Proc. 872, 31-42 (2006).

3. S. Amari and H. Nagaoka, Methods of Information Geometry, Oxford University Press, 2000.

4. C. Cafaro, S. A. Ali and A. Giffin, "An Application of Reversible Entropic Dynamics on Curved Statistical Manifolds", AIP Conf. Proc. 872, 243-251 (2006).

5. C. Cafaro and S. A. Ali, "Jacobi Fields on Statistical Manifolds of Negative Curvature", Physica D (2007), doi: 10.1016/j.physd.2007.07.001.

6. R.A. Fisher, "Theory of statistical estimation" Proc. Cambridge Philos. Soc. 122, 700 (1925).

7. C.R. Rao, "Information and accuracy attainable in the estimation of statistical parameters", Bull. Calcutta Math. Soc. 37, 81 (1945).

8. S. A. Ali and C. Cafaro, "Towards an Information Geometrodynamical Approach to Classical and Quantum Chaos", accepted for presentation at the "Ettore Majorana Centre", Erice-Italy (November, 2007).

9. V.I. Arnold, Mathematical Methods of Classical Physics, Springer-Verlag, 1989.

10. W. H. Zurek and J. P. Paz, "Quantum Chaos: a decoherent definition", Physica D 83, 300 (1995).

11. M. P. do Carmo, Riemannian Geometry, Birkhauser, Boston, 1992.

12. C. W. Misner, K. S. Thorne and J. A. Wheeler, Gravitation, Freeman \& Co., San Francisco, 1973.

13. C. Chicone and B. Mashhoon, "The generalized Jacobi equation", Class. Quantum Grav. 19, (2002).

14. L. Casetti et al., "Riemannian theory of Hamiltonian chaos and Lyapunov exponents", Phys. Rev. E 54, (1996).

15. T. Kawabe, "Indicator of chaos based on the Riemannian geometric approach", Phys. Rev. E 71, (2005).

16. A. J. Scott et al., "Hypersensitivity and chaos signatures in the quantum baker's map", J. Phys. A 39, (2006). 